\title{
Parameter-Free and Multigrid Convergent Digital Curvature Estimators ${ }^{\star}$
}

\author{
Jérémy Levallois ${ }^{1,2}$, David Coeurjolly ${ }^{1}$, and Jacques-Olivier Lachaud ${ }^{2}$ \\ 1 Université de Lyon, CNRS, \\ INSA-Lyon, LIRIS, UMR5205, F-69621, France \\ ${ }^{2}$ Université de Savoie, CNRS, \\ LAMA, UMR5127, F-73776, France
}

\begin{abstract}
In many geometry processing applications, the estimation of differential geometric quantities such as curvature or normal vector field is an essential step. Focusing on multigrid convergent estimators, most of them require a user specified parameter to define the scale at which the analysis is performed (size of a convolution kernel, size of local patches for polynomial fitting, etc). In a previous work, we have proposed a new class of estimators on digital shape boundaries based on Integral Invariants. In this paper, we propose new variants of these estimators which are parameter-free and ensure multigrid convergence in $2 \mathrm{D}$. As far as we know, these are the first parameter-free multigrid convergent curvature estimators.
\end{abstract}

Keywords: Curvature estimation, multigrid convergence, integral invariants, digital straight segments, parameter-free estimators.

\section{Introduction}

Estimating differential quantities like curvatures on discrete data is a tricky task and generally relies on some user supervision to specify some computation window. Indeed, the user has to balance between a small window which preserves most likely sharp features and a big window which offer a better accuracy in flatter smooth zones. Even worse, there may not exist a window size that is appropriate to the whole data. For digital data, another fundamental issue is related to the multigrid convergence property of geometric estimators: this property holds whenever the geometric estimation on a digitized shape is more and more accurate as the digitization step gets finer and finer. It is clear that a user supervision cannot be considered when multigrid convergence is involved. The question is then: can we design parameter-free curvature(s) estimator on digital data ? Furthermore, can this estimator be adaptive to the local data characteristics?

For 2D digital curves, tangent estimation from maximal digital straight segments answers these two questions in a nice way. Indeed, it requires no parameter

\footnotetext{
* This work has been mainly funded by DigitalSnow ANR-11-BS02-009 and KIDICO ANR-2010-BLAN-0205 research grants.
} 
and is proven to be multigrid convergent $7[13$. For curvature estimation, the digitization grid step $h$ is required to get the scale of the shape but is not compulsory to get relative estimations. Two accurate curvature estimators are parameterfree and adaptive: one based on maximal digital circular arcs (MDCA) [12, one based on squared curvature minimization [5. However, their convergence is not proven. To get convergence, authors generally define the computation window as a function of $h$. Binomial or Gaussian convolution estimators 8,4 use some $1 / h^{\alpha}$ window size, digital integral invariants [2] use some $h^{\frac{1}{3}}$ radius size. The polynomial fitting of [1] also requires a thickness parameter. For 3D digital surfaces, the only multigrid convergent estimator of the curvature tensor that we are aware of is the digital integral invariant (II for short) of [2]3; it relies on a $h^{\frac{1}{3}}$ radius size.

This paper proposes a theoretically sound method to get rid of user specified parameters. The idea is to use length properties of maximal segments as functions of $h$ in order to determine automatically a correct computation window. We show how this approach can set automatically the digital radius size required for the multigrid convergence of the II curvature estimator, without any knowledge of the grid step $h$. By this way, we obtain the first parameter-free multigrid convergent curvature estimator for $2 \mathrm{D}$ contours. This approach is also extensible to $3 \mathrm{D}$ by a careful use of axis-aligned slices in the shape. Although we have hints of multigrid convergence, still some work is required to prove it fully.

\section{Preliminaries}

We denote by $Z$ any subset of $\mathbb{Z}^{2}$ (or $\mathbb{Z}^{3}$, depending on the context). In dimension $2, B d(Z)$ denotes the topological boundary of $Z$, seen as a cellular cartesian complex. It is thus composed of $0-$ and 1 -cells (resp. pointels and linels). By convention, we decide to map pointels coordinates to $\mathbb{Z}^{2}$.

Definition 1 (Standard Line and Digital Straight Segment). The set of points $(x, y) \in \mathbb{Z}^{2}$ satisfying $\mu \leq a x-b y<\mu+|a|+|b|$, with $a, b$ and $\mu$ integer numbers, is called the standard digital line with slope $a / b$ and shift $\mu$. Any connected subset of pixels of a standard digital line is a digital straight segment (DSS for short).

Definition 2 (Maximal Segment and Maximal Segment Pencil [7]). A sequence of pointels $\left\{p_{i}, \ldots, p_{j}\right\} \subset B d(Z)$ is a maximal segment iff $\left\{p_{i}, \ldots, p_{j}\right\}$ is a DSS which cannot be extended neither to its front nor to its back while still being a DSS. At a given pointel $p \in B d(Z)$, the pencil of maximal segment at $p$ is the set of maximal segments on $B d(Z)$ containing $p$.

In the multigrid convergence framework, digital objects $Z$ are given by the digitization of a continuous object for a given scale factor $h$. More formally, given a family of Euclidean shapes $\mathbb{X}$, we denote by $\mathrm{D}_{h}(X)$ the Gauss digitization of $X \in \mathbb{X}$ in a $d$-dimensional grid of grid step $h$, i.e.

$$
\mathrm{D}_{h}(X) \stackrel{\text { def }}{=}\left(\frac{1}{h} \cdot X\right) \cap \mathbb{Z}^{d}
$$




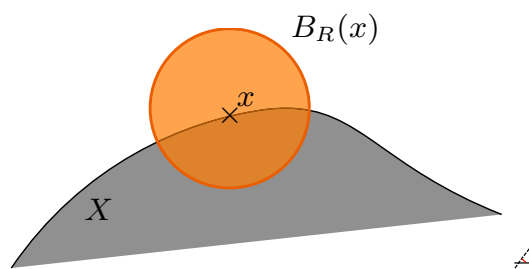

(a)

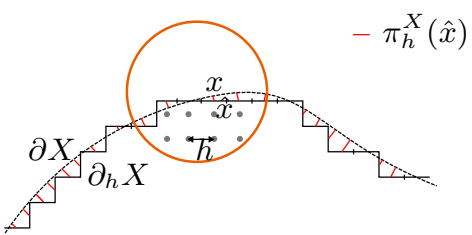

(b)

Fig. 1. Integral invariant computation (left) and notations (right) in dimension 2

Similarly to 3 we denote $\partial_{h} X$ the $h$-boundary of $X$, i.e. a $d$-1-dimensional subset of $\mathbb{R}^{d}$ corresponding to the geometrical embedding of the boundary of the Gauss digitization of $X$ at grid step $h$. In our multigrid convergence framework, quantities are estimated on $\partial_{h} X$ and then compared to associated expected values on $\partial X$ (see Fig. 11). Note that a discrete/combinatorial view of $\partial_{h} X$ is given by $B d(Z)$ with $Z=\mathrm{D}_{h}(X)$. In many situations, maximal segments and maximal segment pencils play a very important role in multigrid digital contour geometry processing 613. For the purpose of this paper, let us focus on the asymptotic properties of lengths of maximal segment:

Lemma 1 (Asymptotic Laws of Maximal Segments [6]). Let $X$ be some convex shape of $\mathbb{R}^{2}$, with at least $C^{3}$-boundary and bounded curvature. The discrete length of maximal segments in $B d(Z)$ for $Z=\mathrm{D}_{h}(X)$ follows:

- the shortest is lower bounded by $\Omega\left(h^{-\frac{1}{3}}\right)$;

- the longest is upper bounded by $O\left(h^{-\frac{1}{2}}\right)$;

- their average length, denoted $L_{D}(Z)$, is such that:

$$
\Theta\left(h^{-\frac{1}{3}}\right) \leq L_{D}(Z) \leq \Theta\left(h^{-\frac{1}{3}} \log \left(\frac{1}{h}\right)\right) .
$$

In [2]3, we have proposed convergent curvature estimators based on Integral Invariants 109 . For short, the idea is to move a geometrical kernel (ball with radius $R$ ) at each surface point and to compute integrals on the intersection between the ball and the digital shape. In dimension 2 , the estimator $\hat{\kappa}_{R}(Z, \hat{x}, h)$ is defined as a function of the number of grid points in $(h \cdot Z) \cap B_{R}(\hat{x}), B_{R}(\hat{x})$ being the ball with radius $R$ centered at $\hat{x}$. In dimension 3 , mean curvature estimation can be obtained from the number of points in $(h \cdot Z) \cap B_{R}(\hat{x})$. Instead of simply estimating the measure (volume) of $X \cap B_{R}(x)$ by discrete summation, we estimate in 3D the covariance matrix of $(h \cdot Z) \cap B_{R}(\hat{x})$. Its eigenvalues/eigenvectors give us quantitative and directional information from which we can design estimators $\hat{\kappa}_{R}^{1}(Z, \hat{x}, h)$ and $\hat{\kappa}_{R}^{2}(Z, \hat{x}, h)$ of principal curvatures $\kappa^{1}$ and $\kappa^{2}$ for $x \in \partial X$. Their multigrid convergence properties are summed up below.

Theorem 1 (Uniform multigrid convergence of $\hat{\kappa}_{R}, \hat{\kappa}_{R}^{1}$, and $\hat{\kappa}_{R}^{2}[\mathbf{2}, \mathbf{3}]$ ). Let $X$ be some convex shape in $\mathbb{R}^{2}$ or $\mathbb{R}^{3}$, with at least $C^{3}$-boundary and bounded 
curvature. Then, there exist positive constants $h_{0}, k$ and $K$, for any $h \leq h_{0}$, setting $R=k h^{\frac{1}{3}}$, we have: $\forall x \in \partial X, \forall \hat{x} \in \partial_{h} X,\|\hat{x}-x\|_{\infty} \leq h$,

$$
\left|\hat{\kappa}_{R}\left(\mathrm{D}_{h}(X), \hat{x}, h\right)-\kappa(X, x)\right| \leq K h^{\frac{1}{3}}
$$

and more specifically for $X$ in $\mathbb{R}^{3}, \forall i \in\{1,2\}$ :

$$
\left|\hat{\kappa}_{R}^{i}\left(\mathrm{D}_{h}(X), \hat{x}, h\right)-\kappa^{i}(X, x)\right| \leq K h^{\frac{1}{3}}
$$

A key point in Theorem 1 is that the radius $R$ of the ball has to be in $\Theta\left(h^{\frac{1}{3}}\right)$ to get the convergence result. In the following, we use geometrical characteristics of $B d(Z)$ (its maximal segment length distribution) to automatically select the appropriate local or global radius $R$ while keeping the multigrid convergence property.

\section{Multigrid Convergence of 2D Parameter-Free Curvature}

Let us first define our new curvature estimator on digital objects $Z \subset \mathbb{Z}^{2}$ :

Definition 3. Given $Z \subset \mathbb{Z}^{2}$, the parameter-free digital curvature estimator $\hat{\kappa}^{*}$ at a pointel $p \in B d(Z)$ is defined as:

$$
\hat{\kappa}^{*}(Z, p) \stackrel{\text { def }}{=} \frac{3 \pi}{2 \rho(Z)}-\frac{3 A(Z, p)}{\rho(Z)^{3}}
$$

where $\rho(Z)=L_{D}^{2}(Z)$ and $A(Z, p)=\operatorname{Card}\left(B_{\rho(Z)}(p) \cap Z\right)$.

To rephrase the definition, we first compute the average discrete length of all maximal segments of $B d(Z)$. Then the symbol $\rho$ is the square of this length. The estimation $\hat{\kappa}^{*}(Z, p)$ is a function of the number of digital points in $Z$ intersected with the ball of radius $\rho$ centered at $p$. In the following, we fill the gap between the parameter-free estimator $\hat{\kappa}^{*}$ and 2D II curvature estimator as described in [2].

First of all, the multigrid convergence framework implies we have a scale factor $h$ between maximal segment lengths on $B d(Z)$ and distances in the Euclidean space on which $\partial_{h} X$ is defined. Hence, for $Z=\mathrm{D}_{h}(X)$, inserting $\rho(Z)=L_{D}^{2}(Z)$ into Lemma 1 implies

$$
\Theta\left(h^{\frac{1}{3}}\right) \leq h \rho(Z) \leq \Theta\left(h^{\frac{1}{3}} \log ^{2}\left(\frac{1}{h}\right)\right) .
$$

In [2]3, we have define a 2D II digital curvature estimator $\hat{\kappa}_{R}$ that depends on a ball radius $R$. Its multigrid convergence is guaranteed whenever the radius is in $\Theta\left(h^{\frac{1}{3}}\right)$. The quantity $\rho(Z)$ relies only on the digital contour geometry of $Z$. Except for the $\log ^{2}(\cdot)$ term, $h \rho(Z)$ is thus an excellent candidate for parameter $R$, since it follows approximately $\Theta\left(h^{\frac{1}{3}}\right)$, to design a parameter free curvature estimator. 
Theorem 2 (Uniform convergence of curvature estimator $\hat{\kappa}^{*}$ ). Let $X$ be some convex shape of $\mathbb{R}^{2}$, with at least $C^{3}$-boundary and bounded curvature. Let $Z=\mathrm{D}_{h}(X)$. Then, there exist a positive constant $h_{0}$, for any $0<h \leq h_{0}$, we have, $\forall x \in \partial X$ and $\forall p \in B d(Z)$

$$
\|h p-x\|_{\infty} \leq h \Rightarrow\left|\frac{1}{h} \hat{\kappa}^{*}(Z, p)-\kappa(X, x)\right| \leq O\left(h^{\frac{1}{3}} \log ^{2}\left(\frac{1}{h}\right)\right) .
$$

Note that $p \in B d(Z)$ implies $h p \in \partial_{h} X$. The parameter-free curvature is rescaled by $h$ in order to compare comparable shapes.

Proof. First, we expand $\frac{1}{h} \hat{\kappa}^{*}(Z, p)$ as

$$
\begin{aligned}
\frac{1}{h} \hat{\kappa}^{*}(Z, p) & =\frac{3 \pi}{2 h \rho(Z)}-\frac{3 A(Z, p)}{h \rho(Z)^{3}}=\frac{3 \pi}{2 h \rho(Z)}-\frac{3 \operatorname{Card}\left(B_{\rho(Z)}(p) \cap \mathrm{D}_{h}(X)\right)}{h \rho(Z)^{3}} \\
& =\frac{3 \pi}{2 h \rho(Z)}-\frac{3 \operatorname{Card}\left(B_{(h \rho(Z)) / h}\left(\frac{1}{h} \cdot(h p)\right) \cap \mathrm{D}_{h}(X), h\right)}{h \rho(Z)^{3}} \\
& =\hat{\kappa}_{R}\left(\mathrm{D}_{h}(X), \hat{x}, h\right) \quad(\text { with } R \stackrel{\text { def }}{=} h \rho(Z) \text { and } \hat{x} \stackrel{\text { def }}{=} h p \text { and [2] }) .
\end{aligned}
$$

It suffices now to bound $\left|\hat{\kappa}_{R}\left(\mathrm{D}_{h}(X), \hat{x}, h\right)-\kappa(X, x)\right|$ according to the asymptotic behavior of $R \stackrel{\text { def }}{=} h \rho(Z)$. According to Eq.(6), $R$ is contained between two bounds:

If $R=\Theta\left(h^{\frac{1}{3}}\right)$, we are in the hypothesis of Theorem 2. so the error term is in $O\left(h^{\frac{1}{3}}\right)$.

If $R=\Theta\left(h^{\frac{1}{3}} \log ^{2}\left(\frac{1}{h}\right)\right)$, we expand the error term in Theorem 2 using Eq. (18) of [2] (with $\alpha^{\prime}=1$ and $\beta=1$ in general case):

$$
\begin{aligned}
& \left|\hat{\kappa}_{R}\left(\mathrm{D}_{h}(X), \hat{x}, h\right)-\kappa(X, x)\right| \\
& \quad \leq O(h \rho(Z))+O\left(\frac{h}{(h \rho(Z))^{2}}\right)+O\left(\frac{h}{(h \rho(Z))^{2}}\right)\left(1+O\left((h \rho(Z))^{2}\right)+O(h)\right) \\
& \quad \leq O\left(h^{\frac{1}{3}} \log ^{2}\left(\frac{1}{h}\right)\right)+O\left(\frac{h^{\frac{1}{3}}}{\log ^{4}\left(\frac{1}{h}\right)}\right)+O(h)+O\left(\frac{h^{\frac{4}{3}}}{\log ^{4}\left(\frac{1}{h}\right)}\right)
\end{aligned}
$$

$O\left(h^{\frac{1}{3}} \log ^{2}\left(\frac{1}{h}\right)\right)$ is the dominant error term in the latter expression. Gathering the two cases and recalling that $\frac{1}{h} \hat{\kappa}^{*}(Z, p)=\hat{\kappa}_{R}\left(\mathrm{D}_{h}(X), \hat{x}, h\right)$, we conclude that the error in Eq.(7) is exactly $O\left(h^{\frac{1}{3}} \log ^{2}\left(\frac{1}{h}\right)\right)$.

The previous curvature estimator is thus convergent. It requires the scale parameter $h$ only to determine the unity used for measuring curvatures. But all curvatures are correct relatively. A possible drawback of the previous estimator is that the ball radius is not adaptive to shape features (for instance sharp features). Instead of using the same ball radius for the whole shape, we can use the maximal segment pencil at each pointel to detect a local size for the radius. We will denote by $\rho(Z, p)$ the square of average length of the maximal segment pencil at pointel $p$. 
Definition 4. Given $Z \subset \mathbb{Z}^{2}$, the local parameter-free curvature estimator $\hat{\kappa}_{l}^{*}$ at a pointel $p \in B d(Z)$ is given by:

$$
\hat{\kappa}_{l}^{*}(Z, p) \stackrel{\text { def }}{=} \frac{3 \pi}{2 \rho(Z, p)}-\frac{3 A^{\prime}(Z, p)}{\rho(Z, p)^{3}}
$$

where $A^{\prime}(Z, p)=\operatorname{Card}\left(B_{\rho(Z, p)}(p) \cap Z\right)$.

In this local version, some maximal segments may have a too long length which prevents us to have multigrid convergence proof. Indeed, if, in the maximal segment pencil, lengths are in the global range of Eq. (2), good multigrid behavior of this local estimator can be expected. Issues arise for maximal segments with longest length in $O\left(h^{-\frac{1}{2}}\right)$ in Lemma 1. In this pathological case, no convergence can be expected. In Sect. 5, we experimentally show very good convergence properties on this estimator.

\section{3D Parameter-Free Curvature Tensor Estimators}

In this section, we present parameter-free curvature tensor estimators in $3 \mathrm{D}$. To sum up, we use the lengths of maximal segments in object slices to automatically set the integral invariant radius parameter. Let us first start with a proposition on smooth manifolds. Let $X$ be any object in $\mathbb{R}^{3}$ with $C^{2}$-smooth boundary $\partial X$ whose absolute principal curvatures are bounded by some constant $K$. The normal to $\partial X$ at $x$ is denoted by $\boldsymbol{n}(x)$. The principal curvatures at $x$ are denoted by $\kappa_{1}(x)$ and $\kappa_{2}(x)$.

Proposition 1. For any $x \in \partial X$, let $\pi_{\boldsymbol{e}}(x)$ be the plane containing $x$ and orthogonal to vector $\boldsymbol{e} \in\{\boldsymbol{x}, \boldsymbol{y}, \boldsymbol{z}\}$. Let $\partial X_{\boldsymbol{e}}(x)$ be the set that is the intersection $X \cap \pi_{\boldsymbol{e}}(x)$. Then at least two of the sets $\partial X_{\boldsymbol{x}}(x), \partial X_{\boldsymbol{y}}(x)$ and $\partial X_{\boldsymbol{z}}(x)$ are locally curves whose curvatures are bounded by $\sqrt{2} K$ in absolute value.

The proof is available in Appendix A.1. The radius $\rho^{\prime}(Z)$ of integral invariant computation will be defined as the square of some average of lengths of maximal segments for a digital object $Z$ in $\mathbb{Z}^{3}$, a more formal definition will be given just after. We may define now our parameter-free curvature estimators in $3 \mathrm{D}$ :

Definition 5. Given $Z \subset \mathbb{Z}^{3}$, the parameter-free mean curvature estimator $\hat{H}^{*}$ at a pointel $p \in B d(Z)$ is defined as:

$$
\hat{H}^{*}(Z, p) \stackrel{\text { def }}{=} \frac{8}{3 \rho^{\prime}(Z)}-\frac{4 V_{\rho^{\prime}(Z)}(Z, p)}{\pi \rho^{\prime}(Z)^{4}},
$$

where $V_{\rho^{\prime}(Z)}(Z, p)=\operatorname{Card}\left(B_{\rho^{\prime}(Z)}(p) \cap Z\right)$.

As discussed in 93, directional curvature information and thus curvature tensor can be estimated from the eigenvalues of the covariance matrix 1 of $\operatorname{Card}\left(B_{\rho^{\prime}(Z)}(p) \cap Z\right)$.

${ }^{1}$ The covariance matrix of $Y \subset \mathbb{R}^{3}$ is defined by $J(Y) \stackrel{\text { def }}{=} \int_{Y}(p-\bar{Y})(p-\bar{Y})^{T} d p$ where $\bar{Y}$ is the centroid of $Y$. 
Definition 6. Let $Z$ be a digital shape in $\mathbb{Z}^{3}$, we define the parameter-free principal curvature estimators $\hat{\kappa}^{1 *}$ and $\hat{\kappa}^{2 *}$ of $Z$ at point $p \in B d(Z)$ as

$$
\hat{\kappa}^{1 *}(Z, p)=\frac{6\left(\hat{\lambda}_{2}-3 \hat{\lambda}_{1}\right)}{\pi \rho^{\prime}(Z)^{6}}+\frac{8}{5 \rho^{\prime}(Z)}, \quad \hat{\kappa}^{2 *}(Z, p)=\frac{6\left(\hat{\lambda}_{1}-3 \hat{\lambda}_{2}\right)}{\pi \rho^{\prime}(Z)^{6}}+\frac{8}{5 \rho^{\prime}(Z)}
$$

where $\hat{\lambda}_{1}$ and $\hat{\lambda}_{2}$ are the two greatest eigenvalues of the covariance matrix of $B_{\rho^{\prime}(Z)}(p) \cap Z$.

Let us now precise what is the $\rho^{\prime}(Z)$ parameter. We provide one global definition $\rho^{\prime}(Z)$ and one local definition $\rho^{\prime}(Z, p)$ for $p \in B d(Z)$ :

Definition 7. Given a digital object $Z$, each surfel $p \in B d(Z)$ is orthogonal to two slices $\pi_{\boldsymbol{e}_{1}(p)}$ and $\pi_{\boldsymbol{e}_{2}(p)}$. For each slice $\pi_{\boldsymbol{e}_{i}(p)} \cap Z$, the pencil of maximal segments covering $p$ determines a set of integers $l_{i}(p)$, formed by the lengths of these maximal segments. Finally, we number by $M(p)$ the slice containing the longest maximal segment (i.e. the slice $i$ whose set $l_{i}(p)$ contains the biggest integer). Then, we define

- $\rho^{\prime}(Z)$ is the square of the average of maximal segment lengths for all slices $\pi_{\boldsymbol{e}_{i}(p)} \cap Z$ of $Z$;

- $\rho^{\prime}(Z, p)$ is the square of the average value of $l_{M(p)}(p)$.

As in 2D, some pathological cases may appear leading to the fact that $h \rho^{\prime}(Z, p) \in$ $\Theta(1)$. In which case, nothing could be expected in terms of multigrid convergence. Again, experimental analysis shows that this bad behavior is not observed. Unlike the $2 \mathrm{D}$ case, we do not have a complete knowledge about the multigrid behavior of $h \rho^{\prime}(Z)$. Let use first express it as a conjecture.

Conjecture 1. Let $X$ be some convex shape of $\mathbb{R}^{3}$, with $C^{3}$-boundary and bounded curvature. Let $Z \stackrel{\text { def }}{=} \mathrm{D}_{h}(X)$, then there exists a positive constant $h_{0}$,

$$
\forall 0<h \leq h_{0}, \quad \Theta\left(h^{\frac{1}{3}}\right) \leq h \rho^{\prime}(Z) \leq \Theta\left(h^{\frac{1}{3}} \log ^{2}\left(\frac{1}{h}\right)\right) .
$$

The rationale behind this conjecture can be sketched as follows. Slicing the objects in all directions, from Proposition 1, we know that at least two third of the slices define convex curves with bounded local curvature information. Since two slices go through one surfel, at least one slice per surfel provides a convex curve with bounded curvature. We thus expect that the lengths of more than half of the maximal segments follow Eq. (13) bounds. Hence, computing the mean of all these lengths provides a stable and consistent quantity which would also follow Eq. (13). In Sect 5. we provide a complete experimental evaluation which supports this conjecture. Assuming Conjecture1, we can prove the two following observations:

Observation 1 (Uniform convergence of $\hat{H}^{*}$.). Let $X$ be some convex shape of $\mathbb{R}^{3}$, with at least $C^{3}$-boundary and bounded curvature. Let $Z \stackrel{\text { def }}{=} \mathrm{D}_{h}(X)$, then 
there exists a positive constant $h_{0}$, for any $0<h \leq h_{0}$, we have $\forall x \in \partial X$ and $\forall p \in B d(Z)$,

$$
\|h p-x\|_{\infty} \leq h \Rightarrow\left|\frac{1}{h} \hat{H}^{*}(Z, p)-H(X, x)\right| \leq O\left(h^{\frac{1}{3}} \log ^{2}\left(\frac{1}{h}\right)\right) .
$$

Assuming Conjecture 1, the proof is similar to the proof of Theorem 2

Observation 2 (Uniform convergence of $\hat{\kappa}^{1 *}$ and $\hat{\kappa}^{2 *}$ ). Let $X$ be some convex shape of $\mathbb{R}^{3}$, with at least $C^{3}$-boundary and bounded curvature. For $i \in$ $\{1,2\}$, recall that $\kappa^{i}(X, x)$ is the $i$-th principal curvature of $\partial X$ at boundary point $x$. Let $Z \stackrel{\text { def }}{=} \mathrm{D}_{h}(X)$, then, there exists a positive constant $h_{0}$, for any $0<h \leq h_{0}$, we have $\forall x \in \partial X$ and $\forall p \in B d(Z)$,

$$
\|h p-x\|_{\infty} \leq h \Rightarrow\left|\frac{1}{h} \hat{\kappa}^{i *}(Z, p)-\kappa^{i}(X, x)\right| \leq O\left(h^{\frac{1}{3}} \log ^{2}\left(\frac{1}{h}\right)\right) .
$$

Proof is available in Appendix A.2.

\section{Experimental Evaluation}

We present an experimental evaluation of our parameter-free curvature estimators described before, in 2D and 3D (mean and principal curvatures). All these estimators are implemented in the open-source $\mathrm{C}++$ library DGtal [1. DGtal provides us a way to construct parametric and implicit 2D and 3D shapes for a given grid step $h$. Furthermore, DGtal holds a collection of estimators and several tools to facilitate the comparison between estimators. In dimension 2 , we compare our estimators with a parameter-free curvature estimator called Mostcentered Digital Circular Arc curvature estimator (MDCA) [12, which gives good results but whose multigrid convergence - although observed - is unfortunately not proven. In dimension 3, there is no parameter-free estimator which provides some multigrid convergence. Therefore, considering an implicit or parametric shape on which the exact curvature is known, we present two different global curvature error measurement for a shape at a given grid step $h$ : the $l_{\infty}$ norm measures the average of the absolute error between estimated and true curvature (it corresponds to the uniform convergence in previous theorems), and the $l_{2}$ norm is the square root of the average of squared errors (it better reflects an average behavior of the estimator).

As described in Sect. 3, we build our estimators by moving a geometrical kernel (an Euclidean ball in $d \mathrm{D}$ ) of radius $h \rho$ in $2 \mathrm{D}$ and $h \rho^{\prime}$ in $3 \mathrm{D}$, and centering it on each surface elements (surfels). The volume or the covariance matrix of the intersection of the kernel and the digital object is then estimated by simple pixel or voxel enumeration. Since the radius of the kernel is $h \rho$ or $h \rho^{\prime}$, we first need to estimate them.

In Fig. 2, we study $h \rho^{\prime}$. We see that experimentally it follows the expected asymptotic behavior of Conjecture 1, i.e. they are bounded between $\Theta\left(h^{\frac{1}{3}}\right)$ and $\Theta\left(h^{\frac{1}{3}} \log ^{2}\left(\frac{1}{h}\right)\right)$. Hence, they define a consistent kernel radius for curvature estimators. In Fig. 3 we present asymptotic error measurements for the proposed 

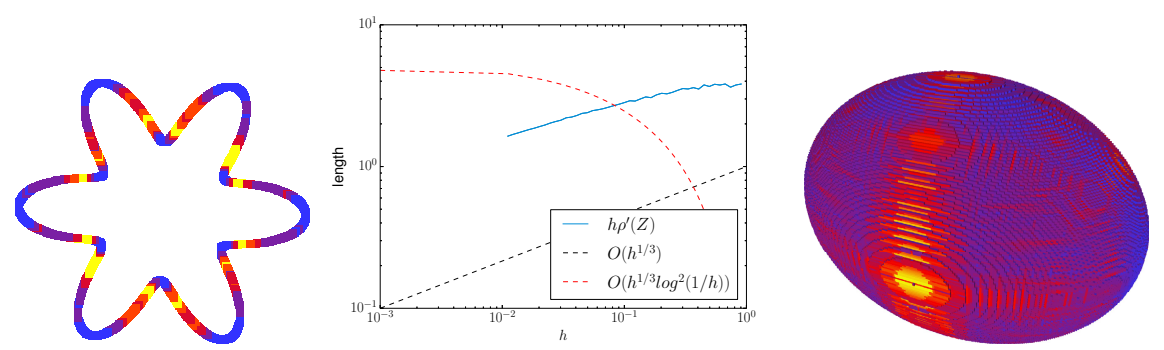

Fig. 2. Clustering of squared length statistics using $K$-means mapped to "flower" contour points (left). Comparison of asymptotic behavior (in log-space) of $h \rho^{\prime}(Z)$ (center) and mapping of length of $h \rho^{\prime}(Z, p)$ on an ellipsoid (right).

parameter-free curvature estimators $\hat{\kappa}^{*}$ and $\hat{\kappa}_{l}^{*}$. These graphs also display error measurements for MDCA [12] and our former non parameter-free version of this estimator $\hat{\kappa}_{R}$ (setting $R=k h^{1 / 3}$ for some constant $k$ ) [2]. We observe that all estimators are convergent with convergence speed at least in $O\left(h^{\frac{1}{3}}\right)$ except for the local parameter-free estimator on the multigrid ellipse.
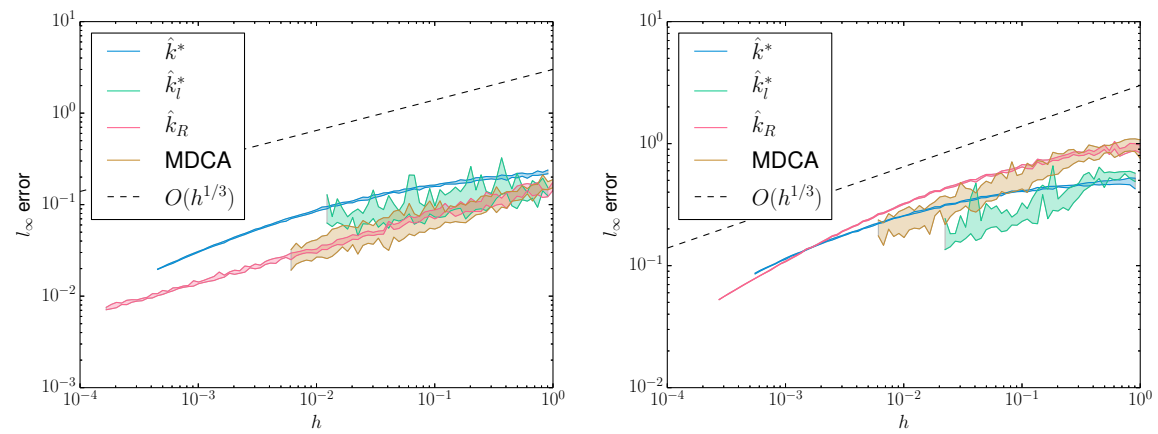

Fig. 3. Comparison in log-space of $l_{\infty}$ curvature error on multigrid ellipses (left) and flowers (right)

In Fig. 4, we present an experimental evaluation of local estimator $\hat{\kappa}_{l}^{*}$ which adapts the kernel size for each point of the digital contour. The main argument for this estimator is to offer an adaptive estimation, for instance to better handle sharp features. As depicted in Fig. [4 for a "flower" shape, we first observe that the local estimator $\hat{\kappa}_{l}^{*}$ has a similar behavior to the global estimator $\hat{\kappa}^{*}$ for the $l_{\infty}$ norm. We also note that, thanks to the adaptive nature of $\hat{\kappa}_{l}^{*}$, the $l_{2}$ error is lower for $\hat{\kappa}_{l}^{*}$ than $\hat{\kappa}^{*}$. For high resolution objects $(i . e$. small $h), \hat{\kappa}_{l}^{*}$ is very time consuming (we need to create a new kernel at each point and we cannot use differential masks as described in [2]). To speed up its computation, we introduce $K$-means variants: we distribute the lengths of maximal segments into $K$ bins by $K$-means clustering, in order to have a limited number of kernels. Hence 


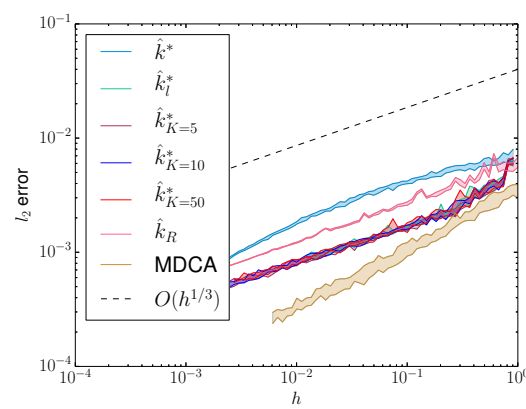

(a)

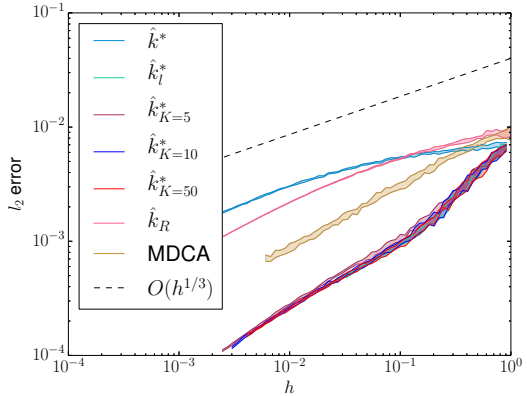

(b)

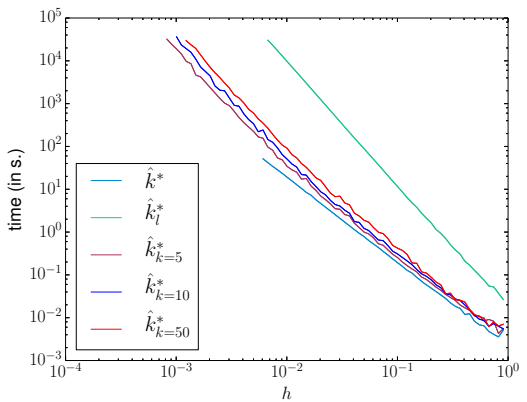

(c)

Fig. 4. Comparison in log-space of $l_{2}$ curvature error on multigrid ellipses (a) and multigrid flowers (b) with the local $\hat{\kappa}_{l}^{*}$ estimator and with different number of precomputed kernels. (c) Computational efficiency of local estimators.
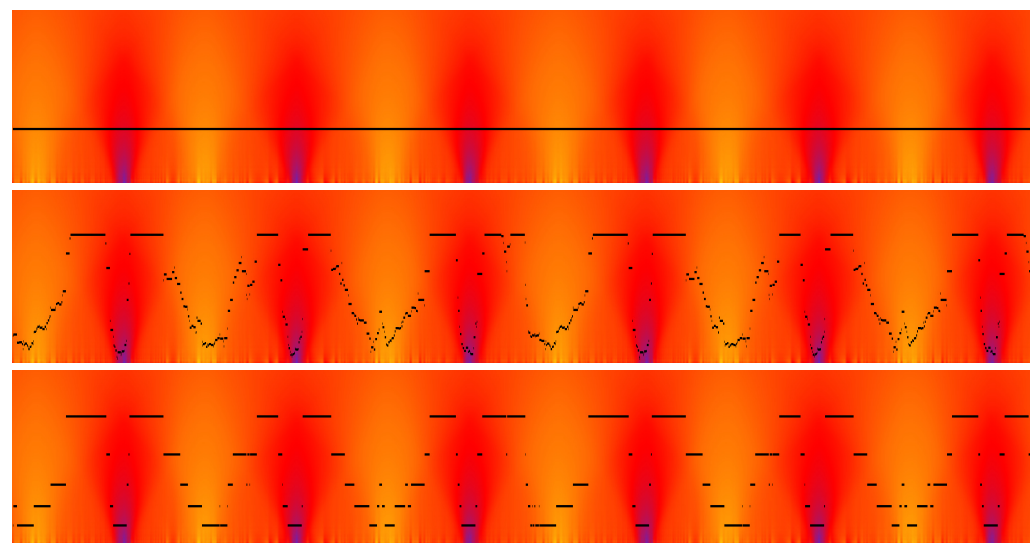

Fig. 5. Curvature scale-space analysis of a flower: $x$-axis is the curvilinear abscissa, $y$-axis is the kernel radius, curvature values are mapped between the blue (lowest curvature) and the yellow color (highest curvature). In black are drawn the radius $\rho(Z)$ for global estimator $\hat{\kappa}^{*}$ (first row), radii $\rho(Z, p)$ for local estimator $\hat{\kappa}_{l}^{*}$ (second row), and radii $\rho(Z, p)$ after $K$-mean clustering for local estimator $\hat{\kappa}_{K=5}^{*}$. (last row) 

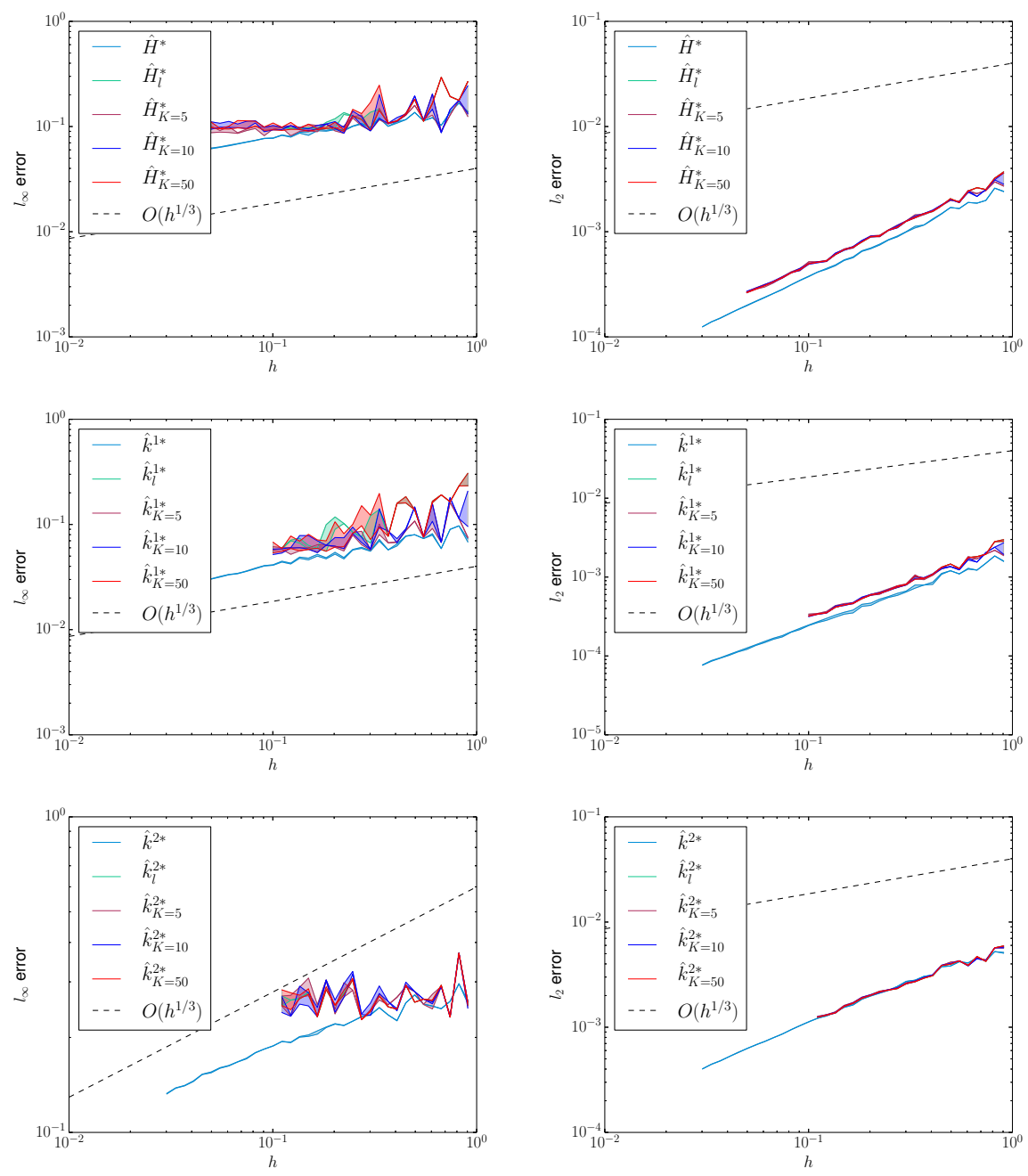

Fig. 6. Comparison in log-space of $l_{\infty}$ (first column) and $l_{2}$ (second column) mean curvature (top), principal curvatures (middle and bottom) errors on a multigrid ellipsoid

mask precomputations are possible. Fig. 2 shows the distribution of $K$-radii in 2D and 3D. In Fig. 4, we tested curvature estimators based on this clustering with $K \in\{5,10,20\}$. We first observe very good multigrid accuracy, even for a small $K$, w.r.t. $\hat{\kappa}_{l}^{*}$ and $\hat{\kappa}^{*}$. In addition, the timing graphs of Fig. $4-(c)$ highlight the interest of considering $K$-means clustering to get an efficient and accurate local estimator. To better understand the local and global length properties of maximal segments, we display on Fig 5 a scale-space view of curvature estimation on the classical "flower" shape and the ball radii respectively used by $\hat{\kappa}^{*}, \hat{\kappa}_{l}^{*}$ and $\hat{\kappa}_{K=5}^{*}$. 

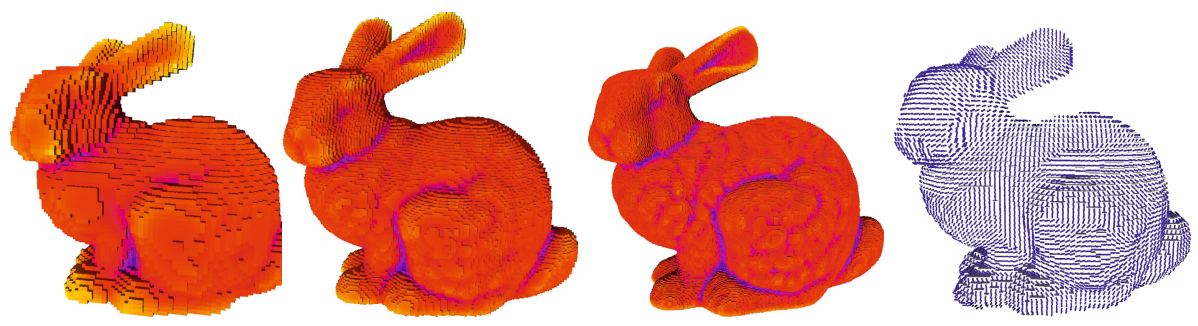

Fig. 7. (Left) Mean curvature mapped on "bunny" at different resolution using $\hat{H}_{l}^{*}$ (yellow color is the highest curvature, blue the lowest). (Right) First principal direction on "bunny" using $\hat{k}_{l}^{*}$ estimator.

Fig. 6 presents 3D result on parameter-free curvature tensor estimators $\hat{H}^{*}$ and $\hat{\kappa}^{i *}$. We also observe the expected $O\left(h^{\frac{1}{3}}\right)$ convergence speed. Similarly to $2 \mathrm{D}$, local estimators (with or without $K$-means clustering) on Fig. 6] (second column) show good multigrid convergence.

\section{Conclusion}

In this article, we have proposed variants of integral invariant estimator to obtain parameter-free curvature estimators in $2 \mathrm{D}$ and $3 \mathrm{D}$. In dimension 2 , we have demonstrated that the parameter-free curvature estimator is also multigrid convergent. As far as we know, this is the first parameter-free curvature estimator with this multigrid property. In dimension 3, we have defined several parameterfree curvature tensor estimators with very good multigrid convergence behaviors. However, convergence proofs rely on an interesting open conjecture on the length distribution of maximal segment in object slices.

\section{References}

1. DGTAL: Digital geometry tools and algorithms library, http://dgtal.org

2. Coeurjolly, D., Lachaud, J.-O., Levallois, J.: Integral based curvature estimators in digital geometry. In: Gonzalez-Diaz, R., Jimenez, M.-J., Medrano, B. (eds.) DGCI 2013. LNCS, vol. 7749, pp. 215-227. Springer, Heidelberg (2013)

3. Coeurjolly, D., Lachaud, J.O., Levallois, J.: Multigrid Convergent Principal Curvature Estimators in Digital Geometry. Computer Vision and Image Understanding (June 2014), http://liris.cnrs.fr/publis/?id=6625

4. Esbelin, H.A., Malgouyres, R., Cartade, C.: Convergence of binomial-based derivative estimation for 2 noisy discretized curves. Theoretical Computer Science 412(36), 4805-4813 (2011)

5. Kerautret, B., Lachaud, J.O.: Curvature estimation along noisy digital contours by approximate global optimization. Pattern Recognition 42(10), 2265-2278 (2009)

6. Lachaud, J.O.: Espaces non-euclidiens et analyse d'image: modèles déformables riemanniens et discrets, topologie et géométrie discrète. Habilitation à diriger des recherches, Université Bordeaux 1, Talence, France (2006) 
7. Lachaud, J.O., Vialard, A., de Vieilleville, F.: Fast, accurate and convergent tangent estimation on digital contours. Image and Vision Computing 25(10), 15721587 (2007)

8. Malgouyres, R., Brunet, F., Fourey, S.: Binomial convolutions and derivatives estimation from noisy discretizations. In: Coeurjolly, D., Sivignon, I., Tougne, L., Dupont, F. (eds.) DGCI 2008. LNCS, vol. 4992, pp. 370-379. Springer, Heidelberg (2008)

9. Pottmann, H., Wallner, J., Huang, Q., Yang, Y.: Integral invariants for robust geometry processing. Computer Aided Geometric Design 26(1), 37-60 (2009)

10. Pottmann, H., Wallner, J., Yang, Y., Lai, Y., Hu, S.: Principal curvatures from the integral invariant viewpoint. Computer Aided Geometric Design 24(8-9), 428-442 (2007)

11. Provot, L., Gérard, Y.: Estimation of the derivatives of a digital function with a convergent bounded error. In: Debled-Rennesson, I., Domenjoud, E., Kerautret, B., Even, P. (eds.) DGCI 2011. LNCS, vol. 6607, pp. 284-295. Springer, Heidelberg (2011)

12. Roussillon, T., Lachaud, J.-O.: Accurate curvature estimation along digital contours with maximal digital circular arcs. In: Aggarwal, J.K., Barneva, R.P., Brimkov, V.E., Koroutchev, K.N., Korutcheva, E.R. (eds.) IWCIA 2011. LNCS, vol. 6636, pp. 43-55. Springer, Heidelberg (2011)

13. de Vieilleville, F., Lachaud, J.O., Feschet, F.: Maximal digital straight segments and convergence of discrete geometric estimators. Journal of Mathematical Image and Vision 27(2), 471-502 (2007)

\section{A Proofs}

\section{A.1 Proof of Proposition 1}

Proof. Since no ambiguity may arise, we remove $(x)$ from all notations. Please also consider Fig. 8 for illustrations. First of all, if one of the $\pi_{\boldsymbol{x}}, \pi_{\boldsymbol{y}}$ and $\pi_{\boldsymbol{z}}$ is the tangent plane at $x$, then the two other planes are normal planes to $\partial X$ at $x$ (they contains the normal $\boldsymbol{n}$ ). In this case, Euler's theorem tells that any curve defined by the intersection of a normal plane and the surface $\partial X$ has a curvature
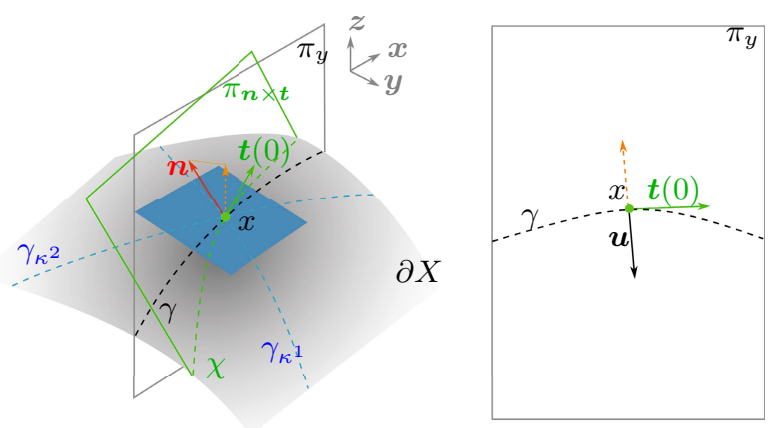

Fig. 8. Notations for Proposition 1 
$\kappa$ equals to $\kappa_{1} \cos ^{2} \theta+\kappa_{2} \sin ^{2} \theta$ for some angle $\theta$. It is then immediate that $|\kappa|$ lies in-between $\left[\min \left(\left|\kappa_{1}\right|,\left|\kappa_{2}\right|\right), \max \left(\left|\kappa_{1}\right|,\left|\kappa_{2}\right|\right)\right]$, and is therefore bounded by $K$ on these two planes.

Otherwise, for each $\boldsymbol{e} \in\{\boldsymbol{x}, \boldsymbol{y}, \boldsymbol{z}\}$, the set $\partial X_{\boldsymbol{e}}$ is locally a $3 \mathrm{D}$ curve that crosses $x$ on the surface $\partial X$. First remark that there is at most one $\boldsymbol{e} \in\{\boldsymbol{x}, \boldsymbol{y}, \boldsymbol{z}\}$ such that $\boldsymbol{n} \cdot \boldsymbol{e} \geq \frac{\sqrt{2}}{2}$. Indeed, let $\boldsymbol{n}=a \boldsymbol{x}+b \boldsymbol{y}+c \boldsymbol{z}$ and for instance $\boldsymbol{n} \cdot \boldsymbol{x} \geq \frac{\sqrt{2}}{2}$, then $b^{2}+c^{2}=1-a^{2}=1-(\boldsymbol{n} \cdot \boldsymbol{x})^{2}<\frac{1}{2}$. Hence both $b=\boldsymbol{n} \cdot \boldsymbol{y}$ and $c=\boldsymbol{n} \cdot \boldsymbol{z}$ are smaller than $\frac{\sqrt{2}}{2}$. We only consider a vector $\boldsymbol{e} \in\{\boldsymbol{x}, \boldsymbol{y}, \boldsymbol{z}\}$ such that $\boldsymbol{n} \cdot \boldsymbol{e} \leq \frac{\sqrt{2}}{2}$.

Let $\chi$ be the curve defined by the intersection of $\partial X$ and the plane $\pi_{\boldsymbol{n} \times \boldsymbol{t}}$ containing $\boldsymbol{n}$ and the tangent $\boldsymbol{t}$ at $x$ of $\partial X_{\boldsymbol{e}}$. From Meusnier's theorem, we have the following relationship between the curvature of $\kappa_{\chi}$ of $\chi$ and the curvature $\kappa$ of $\partial X_{\boldsymbol{e}}$ at $x: \kappa_{\chi}=\kappa \cdot \cos \alpha, \alpha$ being the angle between planes $\pi_{\boldsymbol{e}}$ and $\pi_{\boldsymbol{n} \times \boldsymbol{t}}$. Since $\cos \alpha=\boldsymbol{n} \cdot \boldsymbol{e}$ and $\frac{\sqrt{2}}{2} \leq \cos \alpha \leq 1$, we finally have:

$$
\left|\kappa_{\chi}\right| \leq|\kappa| \leq \sqrt{2}\left|\kappa_{\chi}\right|
$$

Again, since $\left|\kappa_{\chi}\right|$ lies in-between $\left[\min \left(\left|\kappa_{1}\right|,\left|\kappa_{2}\right|\right), \max \left(\left|\kappa_{1}\right|,\left|\kappa_{2}\right|\right)\right]$, it is bounded by $K$ and we have the final result.

\section{A.2 Proof of Uniform Multigrid Convergence of $\hat{\kappa}^{1 *}$ and $\hat{\kappa}^{2 *}$}

Proof. As in 2D, we need to check the convergence of both error bounds of $\rho^{\prime}$. Assuming that Conjecture 1 is true, we have: If $h \rho^{\prime}(Z)=\Theta\left(h^{\frac{1}{3}}\right)$, we are in the hypothesis of Theorem 1, the error term is in $O\left(h^{\frac{1}{3}}\right)$. If $h \rho^{\prime}(Z)=\Theta\left(h^{\frac{1}{3}} \log ^{2}\left(\frac{1}{h}\right)\right)$, we can decompose the error term using Equation (28) of [3] (setting $\mu_{i}=1$ in Eq.(28)):

$$
\begin{aligned}
\left|\frac{1}{h} \hat{\kappa}^{1 *}(Z)-\kappa^{1}(X, x)\right|=\mid & \left|\hat{\kappa}_{R}^{1}\left(\mathrm{D}_{h}(X), \hat{x}, h\right)-\kappa^{1}(X, x)\right| \\
& \leq O(R)+O\left(\frac{h}{R^{2}}\right) \\
& \leq O\left(h^{\frac{1}{3}} \log ^{2}\left(\frac{1}{h}\right)\right)+O\left(\frac{h^{\frac{1}{3}}}{\log ^{4}\left(\frac{1}{h}\right)}\right)
\end{aligned}
$$

The upper bound error term is $O\left(h^{\frac{1}{3}} \log ^{2}\left(\frac{1}{h}\right)\right)$. Proof for $\hat{\kappa}^{2 *}$ follows similarly. Finally, $\Theta\left(h^{\frac{1}{3}}\right) \leq h \rho^{\prime} \leq \Theta\left(h^{\frac{1}{3}} \log ^{2}\left(\frac{1}{h}\right)\right)$ implies $\left|\frac{1}{h} \hat{\kappa}^{i *}(Z)-\kappa^{i}(X, x)\right| \leq$ $O\left(h^{\frac{1}{3}} \log ^{2}\left(\frac{1}{h}\right)\right)$. 